\title{
Triggered star formation in the environment of young massive stars
}

\author{
Matthias Gritschneder ${ }^{1}$, T. Naab ${ }^{1}$, F. Heitsch ${ }^{2}$ and A.Burkert ${ }^{1}$ \\ ${ }^{1}$ Universitäts-Sternwarte München, Scheinerstr. 1, D-81679 München, Germany \\ ${ }^{2}$ Dept. of Astronomy, University of Michigan, Michigan, United States
}

\begin{abstract}
Recent observations with the Spitzer Space Telescope show clear evidence that star formation takes place in the surrounding of young massive O-type stars, which are shaping their environment due to their powerful radiation and stellar winds. In this work we investigate the effect of ionising radiation of massive stars on the ambient interstellar medium (ISM): In particular we want to examine whether the UV-radiation of O-type stars can lead to the observed pillar-like structures and can trigger star formation. We developed a new implementation, based on a parallel Smooth Particle Hydrodynamics code (called IVINE), that allows an efficient treatment of the effect of ionising radiation from massive stars on their turbulent gaseous environment. Here we present first results at very high resolution. We show that ionising radiation can trigger the collapse of an otherwise stable molecular cloud. The arising structures resemble observed structures (e.g. the pillars of creation in the Eagle Nebula, M16, or the Horsehead Nebula, B33). Including the effect of gravitation we find small regions that can be identified as formation places of individual stars. We conclude that ionising radiation from massive stars alone can trigger substantial star formation in molecular clouds.
\end{abstract}

Keywords. stars: formation, ISM: structure, turbulence, ultraviolet: ISM, methods: numerical

\section{Overview}

In the surroundings of hot OB-Associations filamentary substructures on different scales are observed (see e.g. Sugitani et al. (2002) and references therein). As observational resolution increases, more and more sub-millimeter sources, which could trace the birth of future stars, are detected (e.g. Ward-Thompson et al. 2006). It has long been suggested that radiation driven implosion of molecular clouds can explain the morphology and the star formation in these regions (e.g. Elmegreen et al. 1995).

Recent simulations (see e.g. Mellema et al. (2006), Dale et al. (2005), Kessel-Deynet \& Burkert (2003)) demonstrate the importance of massive stars for the subsequent evolution of their parental molecular clouds. The ionising radiation is a vital ingredient to understand the disruption of molecular clouds and their star formation efficiency.

Our goal is to investigate the morphology of molecular clouds and the formation of protostars in much greater detail. To do so we use very high resolution simulations of a small region of a molecular cloud ionised by a massive nearby star.

\section{Numerical method}

We use the prescription for ionising UV-radiation of a young massive star proposed by Kessel-Deynet \& Burkert (2000). The ionisation degree $x$ is related to the hydrodynamical quantities by an approximation for the resulting temperature of a partly ionised gas

$$
T=T_{\text {ion }} \cdot x+T_{\text {cold }} \cdot(1-x) \text {. }
$$




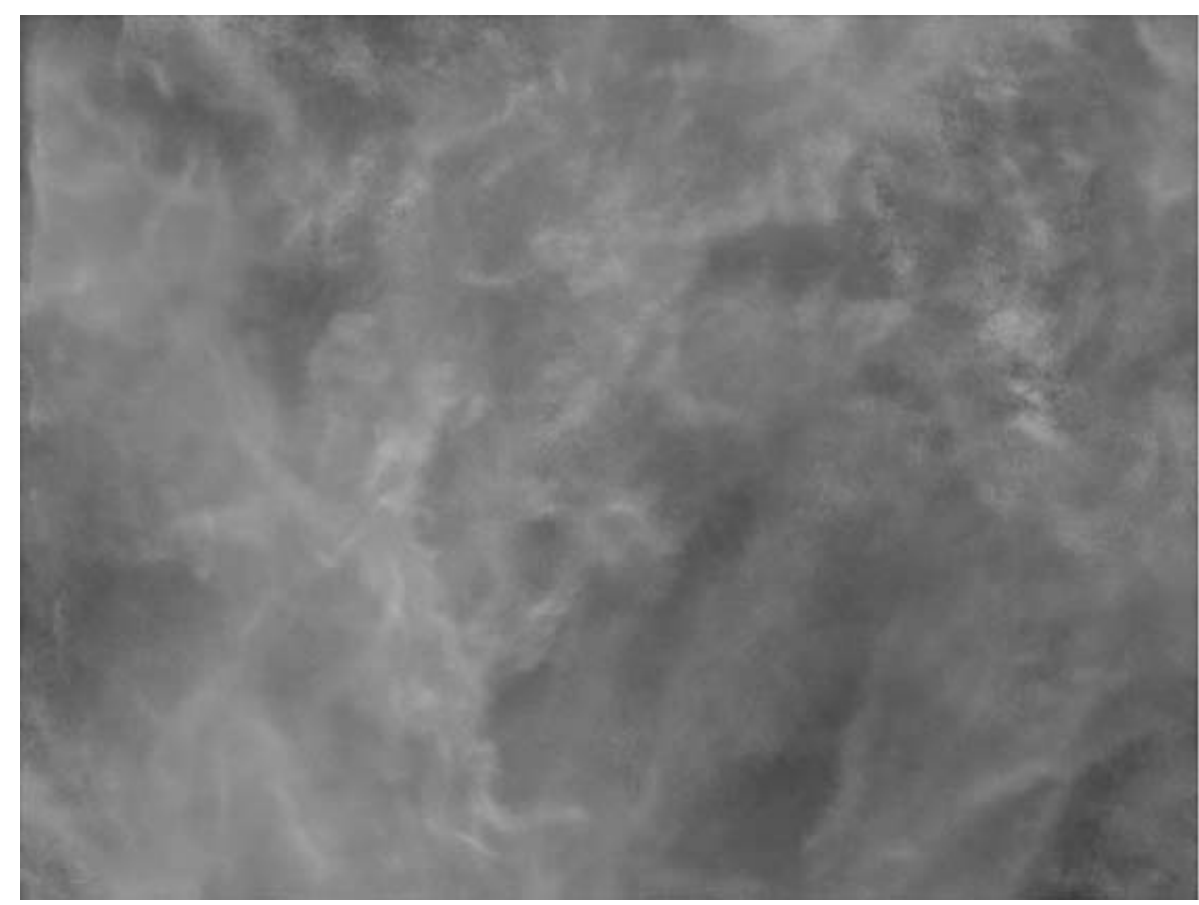

Figure 1. Initial density distribution after the turbulence has decayed to Mach 5 . The box dimension are $2 \mathrm{pc}$ in each direction.

$T_{\text {cold }}$ is the initial temperature of the cold, un-ionised gas and $T_{i o n}$ is the temperature of the ionised gas.

To treat the hydrodynamic and gravitational evolution we use a parallel smoothed particle hydrodynamics (SPH) code called VINE (Wetzstein et al., in prep.). Its Lagrangian nature renders it extremely adept to cover several orders of magnitude in density and time, which is important to follow local gas collapse. We assume plane-parallel UVirradiation of the simulated area, mimicking a radiation source sufficiently far away such that its distance is larger than the dimensions of the area of infall. To couple ionisation to hydrodynamics we use a flux conserving ray-shooting algorithm. A two dimensional grid is superimposed on the area of interest. Along each of the thereby created bins, the optical depth is calculated. The size of each bin, i.e. the grid resolution, is defined by the volume each SPH-particle occupies. This guarantees that the density information given by the SPH-formalism is transformed to the calculation of radiation correctly.

This implementation is fully parallelised. We call it IVINE (Ionisation+VINE).

\section{Numerical tests}

A standard test for numerical implementations of ionizing radiation has been proposed by Lefloch \& Lazareff (1994). It deals with the steady propagation of an ionisation front: a box of constant density is exposed to a time-dependent ionising source. The initial conditions were chosen to be $n_{0}=100 \mathrm{~cm}^{-3}$ and $T_{\text {cold }}=100 \mathrm{~K}$ to enable a direct comparison to their results. The flux increases linearly with time, starting at zero: $\mathrm{d} J / \mathrm{d} t=5.07 \cdot 10^{-8}$ $\mathrm{cm}^{-2} \mathrm{~s}^{-2}$. The recombination parameter $\alpha_{B}$ is set to $\alpha_{B}=2.7 \cdot 10^{-13} \mathrm{~cm}^{3} \mathrm{~s}^{-1}$. The ionised temperature is $T_{i o n}=10^{4} \mathrm{~K}$. At the beginning a small fraction of the box is ionised. Due 


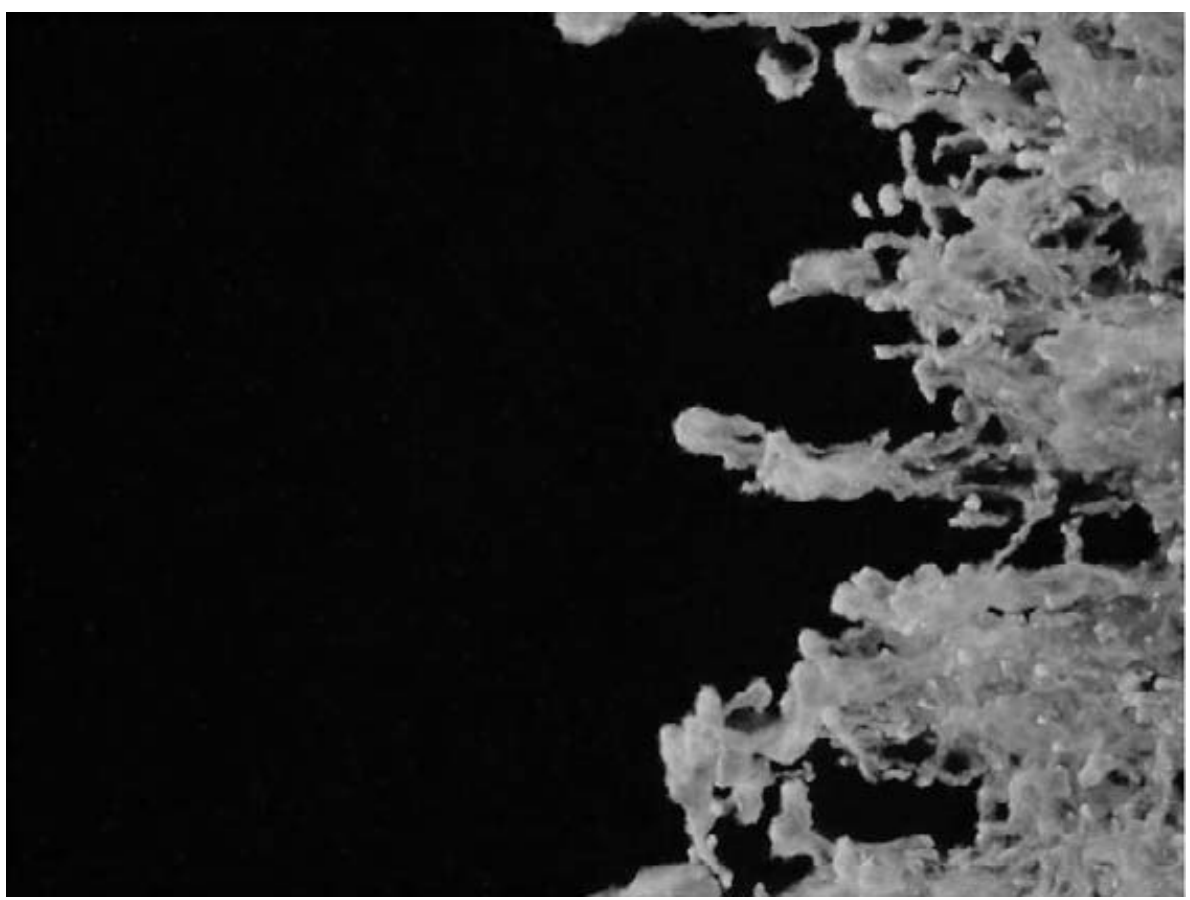

Figure 2. Final stage of evolution after $t \approx 300$ kyrs: the morphology has clearly evolved. The size of the most prominent pillar is roughly $1 \mathrm{pc}$ as it is observed in e.g. M16

Table 1. Comparison of analytical and numerical results for the Lefloch test.

\begin{tabular}{ccccc}
\hline & Analytical & Lefloch \& Lazareff (1994) & Kessel-Deynet \& Burkert (2000) & IVINE \\
\hline$n_{c}\left(\mathrm{~cm}^{-3}\right)$ & 159 & 169 & 155 & 156.8 \\
$n_{i}\left(\mathrm{~cm}^{-3}\right)$ & 0.756 & 0.748 & 0.75 & 0.756 \\
$v_{i}\left(\mathrm{~km} \mathrm{~s}^{-1}\right)$ & 3.48 & 3.36 & 3.43 & 3.41 \\
$v_{s}\left(\mathrm{~km} \mathrm{~s}^{-1}\right)$ & 3.71 & 3.51 & 3.67 & 3.63 \\
\hline
\end{tabular}

to the higher temperature of the ionised gas a shock front evolves. This shock front moves at a constant speed through the box. The analytical solution provides the exact position and speed of the front as well as of the ionised gas at any given time. We find a very good agreement with the analytical solution, the results are shown in Table 1. $n_{c}$ and $n_{i}$ denote the number density of the compressed layer and of the ionised gas, $v_{i}$ and $v_{s}$ are the velocities of the ionisation front and the shock front respectively.

\section{First application: ionisation of a turbulent ISM}

\subsection{Initial conditions}

The first high resolution simulations we performed with the new code address the effect of ionising radiation on a box of turbulent medium. We choose the initial conditions to mimic observed turbulence in the ISM. The cubic simulation domain with a volume of $(2 \mathrm{pc})^{3}$ and a mean density of $\bar{n}=100 \mathrm{~cm}^{-3}$ is set up with a temperature of $T_{\text {cold }}=10 \mathrm{~K}$. The turbulent velocity field is set up adapting a Gaussian random field with a steep power spectrum. The velocity field generates density fluctuations and after a dynamical timescale a turbulent medium with typical velocities of Mach 5 has been generated (see 
Fig. 1). At this point the box is exposed to ionising radiation. The UV-radiation is impinging from the negative $\mathrm{x}$-direction with a flux $J=8.36 \cdot 10^{8} \mathrm{~cm}^{-2} \mathrm{~s}^{-1}$. This leads to a rapid ionisation of the first $\approx 5 \%$ of the cube before the medium reacts to the increased temperature of the ionised gas. The simulations were performed with 2 Million particles on a SGI Altix supercomputer.

\subsection{Results}

The UV-radiation traces the turbulent density distribution, reaching further into the low density regions, and less far in the regions of high density. After a dynamical timescale the hydrodynamics react to the increase in temperature, shock fronts evolve and compress the gas while at the same time increasing the turbulent energy of the cold gas. During this phase a typical morphology evolves. The denser regions shadow regions behind them whereas in lower density regimes the radiation can propagate much further. After the first phase of maximum compression a more quiescent phase of evaporation sets in. The densities are not as high as before, but the structures become even more clear. This leads in the final stage to filamentary, pillar-like substructures, pointing towards the source of radiation as can be seen in Fig. 2. The structures contain high density regions in their tips. These are very likely to become gravitational unstable after a free fall time.

\section{Conclusion}

We developed a fully parallel treatment for the ionising radiation of young massive stars. Ionising Radiation alone is sufficient to explain the morphology observed in the surroundings of hot OB-clusters. In our simulations of turbulent ISM exposed to UVradiation characteristic trunks similar to the ones observed in M16 evolve. Further studies including gravity will show whether the UV-radiation from young massive stars is sufficient to trigger gravitational collapse within these pillars.

\section{Acknowledgements}

M. Gritschneder is supported by the Sonderforschungsbereich 375-95 Astro-ParticlePhysics of the Deutsche Forschungsgemeinschaft.

\section{References}

Dale, J.E., Bonnell, I.A., Clarke, C.J. \& Bate, M.R. 2005, MNRAS 358, 291

Elmegreen, B.G., Kimura, T. \& Tosa, M. 1995, ApJ 451, 675

Kessel-Deynet, O. \& Burkert, A. 2000, MNRAS 315, 713

Kessel-Deynet, O. \& Burkert, A. 2003, MNRAS 338, 545

Lefloch, B. \& Lazareff, B. 1994, A\& A 289, 559

Mellema, G., Arthur, S.J., Henney, W.J., Iliev, I.T. \& Shapiro, P.R. 2006, ApJ 647, 397

Sugitani, K., Tamura, M., Nakajima, Y., Nagashima, C., Nagayama, T., Nakaya, H., Pickles, A.J., Nagata, T.,Sato, S., Fukuda, N. \& Ogura, K. 2002, ApJ 565, L25

Ward-Thompson, D., Nutter, D., Bontemps, S., Whitworth, A. \& Attwood, R. 2006, MNRAS 369,1201

\section{Discussion}

Deharveng: To compare your simulations with the observations, for example of the pillars in the Eagle Nebula, you must look at: 1. the morphology of your structures, 2 . the mass contains in these structures, and 3. the life-time of these structures (you need time to form the young low-mass stars observed inside these structures). Point 1 is very convincing, but what about points 2 or 3 ? 
Gritschneder: On 2: the average mass per pillar is roughly $1 \mathrm{M}_{\odot}$, the whole box initially contains $20 \mathrm{M}_{\odot}$. on 3: the structures evolve at $\sim 300 \mathrm{kyr}$ (the end of the movie I showed) and stay stable until the end of our simulation of $600 \mathrm{kyr}$. It is hard to tell if this is sufficiently long enough since the free fall time at this density is $\sim 160 \mathrm{kyr}$, so it is on the same timescale. The simulations with gravity will show the answer to this question. 